\title{
Synthetic auxotrophy remains stable after continuous evolution and in co-culture with
} mammalian cells

3 Authors: Aditya M. Kunjapur ${ }^{1,2 \dagger *}$, Michael G. Napolitano ${ }^{1,3 \dagger}$, Eriona Hysolli ${ }^{1 \dagger}$, Karen

4 Noguera $^{1}$, Evan M. Appleton ${ }^{1}$, Max G. Schubert ${ }^{1}$, Michaela A. Jones ${ }^{2}$, Siddharth Iyer ${ }^{4}$, Daniel J.

$5 \quad$ Mandell ${ }^{1,5} \&$ George M. Church ${ }^{1 *}$

\section{Affiliations:}

${ }^{1}$ Department of Genetics, Harvard Medical School, 77 Avenue Louis Pasteur, NRB 238, Boston, MA 02115, USA.

${ }^{2}$ Present Address: Department of Chemical and Biomolecular Engineering, University of Delaware, 150 Academy Street, CLB 215, Newark, DE 19716, USA

${ }^{3}$ Present Address: Ginkgo Bioworks, 27 Drydock Avenue, $8^{\text {th }}$ Floor, Boston, MA 02210, USA. ${ }^{4}$ Present Address: Johns Hopkins University, 3101 Wyman Park Drive, Baltimore, MD 21218 , USA

${ }^{5}$ Present Address: GRO Biosciences, 700 Main Street North, Cambridge, MA 02139, USA.

*Corresponding authors: AMK (kunjapur@udel.edu) or GMC

(gchurch@genetics.med.harvard.edu).

$\dagger$ These authors contributed equally to this work.

\section{Abstract:}

Understanding the evolutionary stability and possible context-dependence of biological containment techniques is critical as engineered microbes are increasingly under consideration for applications beyond biomanufacturing. While batch cultures of synthetic auxotrophic Escherichia coli previously exhibited undetectable escape throughout 14 days of monitoring, the long-term effectiveness of synthetic auxotrophy is unknown. Here, we report automated continuous evolution of a synthetic auxotroph using custom chemostats that supply a decreasing concentration of essential biphenylalanine (BipA). After 100 days of evolution in three separate trials, populations exhibit no observable escape and are capable of normal growth rates at 10-fold lower BipA concentration than the ancestral synthetic auxotroph. Allelic reconstruction of three proteins implicated in small molecule transport reveals their contribution to increased fitness at 
30 low BipA concentrations. Mutations do not appear in orthogonal translation machinery nor in

31 synthetic auxotrophic markers. Based on its evolutionary stability, we introduce the progenitor

32 synthetic auxotroph directly to mammalian cell culture. We observe containment of bacteria

33 without detrimental effects on HEK293T cells. Overall, our findings reveal that synthetic

34 auxotrophy is effective on timescales and in contexts that enable diverse applications.

35

\section{One Sentence Summary:}

37 To ascertain whether life inevitably finds a way, we continuously evolve an Escherichia

38 coli strain that was not able to escape from engineered biocontainment before, and we find that it

39 does not escape even after 100 days of evolution, nor does it escape when added to mammalian

40 cell culture. 


\section{Introduction:}

New safeguards are needed for the deliberate release of engineered microbes into the environment, which has promise for applications in agriculture, environmental remediation, and medicine ${ }^{1}$. Genetically encoded biocontainment strategies enable attenuation of engineered live bacteria for diverse biomedical applications ${ }^{2-4}$, including as potential vaccines ${ }^{5-10}$, diagnostics ${ }^{11}$, and therapeutics ${ }^{12-15}$. Auxotrophy, which is the inability of an organism to synthesize a compound needed for its growth, is an existing strategy for containment. However, foundational studies of auxotrophic pathogens demonstrated proliferation in relevant biological fluids ${ }^{16}$ and reversion to prototrophy upon serial passaging ${ }^{17,18}$. Modern genome engineering strategies can prevent auxotrophic reversion, and auxotrophy has been a key component of microbial therapies that have reached advanced clinical trials. However, the ability for auxotrophs to access required metabolites within many host microenvironments, and after leaving the host, remains unaddressed. Auxotrophy may not be effective in scenarios where engineered living bacteria encounter metabolites from dead host cells ${ }^{19}$ or invade host cells ${ }^{20}$. Indeed, growth of double auxotrophs is supported in vivo by neoplastic tissue ${ }^{13}$. Auxotrophy may also be insufficient for tight control of cell proliferation in environments rich with microbial sources of cross-feeding ${ }^{21}$, such as gut, oral, skin, and vaginal microbiomes. Given that most naturally occurring microorganisms are auxotrophs ${ }^{22}$, it is also unlikely that auxotrophy will limit the spread of an engineered microbe once it leaves the body and enters the environment.

Synthetic auxotrophy may overcome these hurdles by requiring provision of a synthetic molecule for survival of the engineered bacteria. This strategy was first implemented successfully in E. coli by engineering an essential protein to depend on incorporation of a nonstandard amino acid (nsAA) ${ }^{23,24}$. We previously engineered $E$. coli strains for dependence on the nsAA biphenylalanine (BipA) by computer-aided redesign of essential enzymes in conjunction with expression of orthogonal translation machinery for BipA incorporation ${ }^{23}$. Among several synthetic auxotrophs originally constructed, one strain harbored three redesigned, nsAAdependent genes: Adenylate kinase ( $a d k . d 6)$, tyrosyl-tRNA synthetase (tyrS.d8), and BipAdependent aminoacyl-tRNA synthetase, for aminoacylation of BipA (BipARS.d6). This BipAdependent strain, dubbed "DEP", exhibited undetectable escape throughout 14 days of monitoring at an assay detection limit of $2.2 \times 10^{-12}$ escapees per colony forming unit (CFU) ${ }^{23}$. 
Though this strain demonstrates effective biocontainment in $1 \mathrm{~L}$ batch experiments, its precise escape frequency and long-term stability remained unexplored.

Here, we perform the first study of evolutionary stability of a synthetic auxotroph, with the aid of automated continuous evolution. Continuous evolution better emulates scenarios where biocontainment may be needed by fostering greater genetic variability within a population. We posited that decreasing BipA concentrations would add selective pressure for adaptation or for escape, either of which would be enlightening. Adaptive laboratory evolution of DEP may improve its fitness in relevant growth contexts, as previously demonstrated for its nonauxotrophic but recoded ancestor, $\mathrm{C} 321 . \Delta \mathrm{A}^{25}$. We report that DEP maintains its inability to grow in the absence of synthetic nutrient, even after three parallel 100-day chemostat trials. Additionally, we find evidence of adaptation, with evolved DEP isolates requiring 10-fold lower BipA concentration to achieve optimal growth than ancestral DEP $(0.5 \mu \mathrm{M}$ rather than $5 \mu \mathrm{M})$. We resequence evolved populations and perform allelic reconstruction in ancestral DEP using multiplex automatable genome engineering (MAGE), identifying alleles that partially restore the adaptive phenotype. Finally, we advance this technology towards host-microbe co-culture applications, demonstrating direct mixed culture of DEP and mammalian cells without need for physical barriers nor complex fluidics.

\section{Results:}

\section{Continuous evolution}

To perform continuous evolution of E. coli, we constructed custom chemostats for parallelized and automated culturing (Fig. 1A). Our design and construction was based on the eVOLVER system ${ }^{26}$, an open-source, do-it-yourself automated culturing platform (Figs. S1-S4). By decreasing BipA concentration over time in our chemostats, we provide an initial mild selection for escape and steadily increase its stringency. This design is analogous to a "morbidostat", where a lethal drug is introduced dynamically at sub-lethal concentrations to study microbial drug resistance ${ }^{27}$, but with synthetic auxotrophy providing selective pressure. Our working algorithm for automated adjustment of BipA concentration as a function of turbidity is shown in Fig. 1B, and a representative image of our hardware is shown in Fig. 1C. 

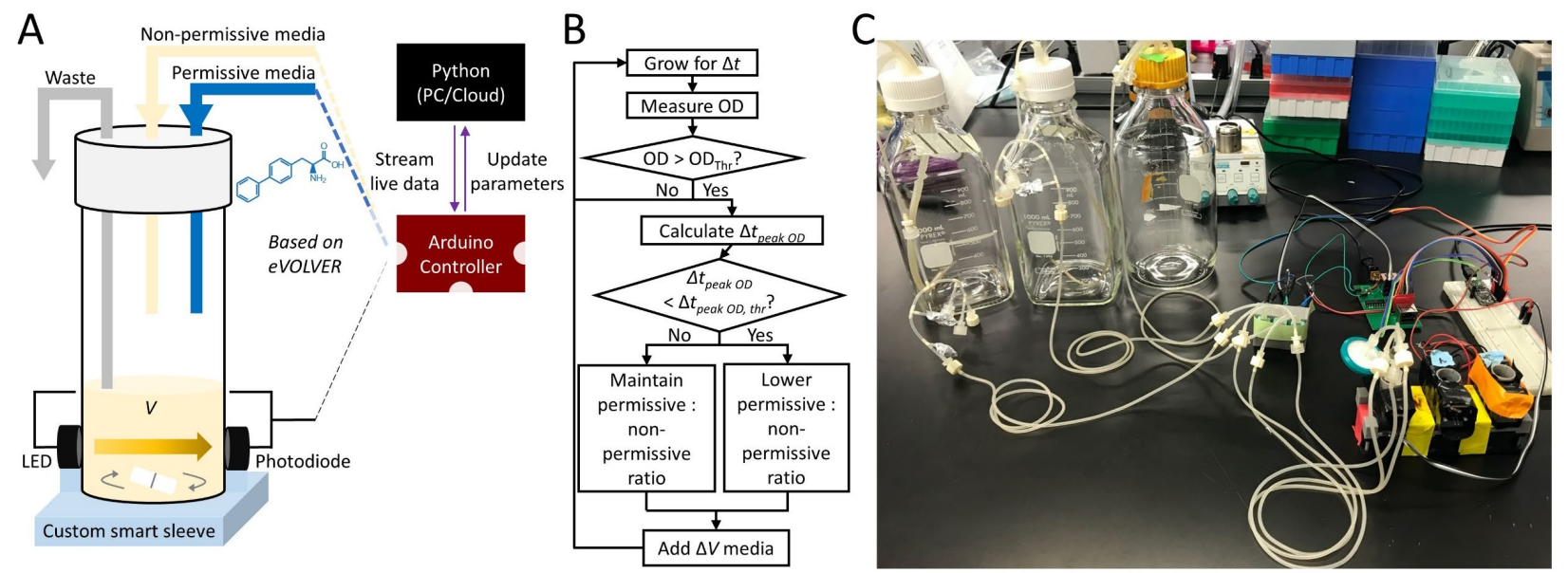

Figure 1. Design and operation of custom chemostats for automated continuous evolution. (A) Illustration of a smart sleeve connected to separate non-permissive media and biphenylalanine (BipA, structure shown in blue) feed lines for automated adjustment of BipA concentration based on growth rate. Pumps and optics are integrated with Arduino controller hardware and Python software based on the eVOLVER do-it-yourself automated culturing framework. (B) Working algorithm for maintenance of cultures in continuous evolution mode. Criteria for lowering the BipA concentration is based on the difference in time elapsing between OD peaks $\left(\Delta t_{\text {peak }} O D\right)$. Smaller time elapsed between OD peaks is indicative of higher growth rates, triggering decrease of BipA concentration when below a threshold value. (C) Representative configuration of hardware for parallelized evolution in triplicate, with three empty sleeves shown.

We inoculated one chemostat with DEP for an 11-day incubation at constant BipA concentration $(100 \mu \mathrm{M})$ (Fig. 2A). After observing no colony formation on non-permissive media, we then inoculated three chemostats in parallel where BipA supply decreased automatically over the following 90 days from $100 \mu \mathrm{M}$ to nearly $10 \mathrm{nM}$ (Fig. 2B). To determine whether the decrease in BipA supply was due to escape from dependence on BipA, we periodically performed escape assays. We continued to observe no escape, including when we seeded liter-scale cultures and plated the associated outgrowth on non-permissive media. Evolved isolates were obtained after this procedure, and their growth was characterized across BipA concentrations (Fig. 2C). At 0.5-1 $\mu \mathrm{M}$ BipA, we observed growth of all evolved isolates, and no growth of the ancestral DEP strain.

\section{Genome resequencing and allelic reconstruction}

To identify the causal alleles contributing to decreased BipA requirement of all three evolved isolates, we performed whole-genome sequencing and mutational analysis. We expected that mutations in auxotrophic markers or orthogonal translation machinery associated with aminoacylation of BipA would be observed. However, no variants were detected in the plasmidexpressed orthogonal translation machinery (aminoacyl-tRNA synthetase and tRNA) reference sequence. Instead, in all three evolved isolates, variants were observed in three non-essential 

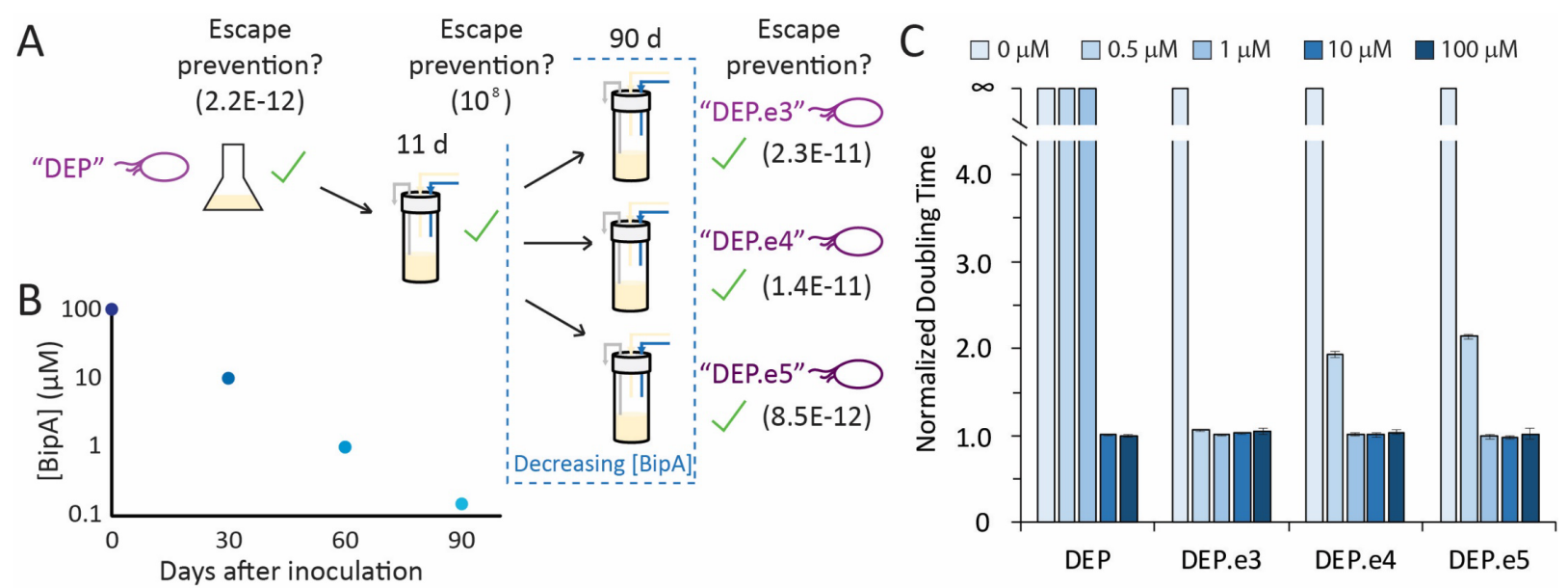

Figure 2. Continuous evolution of synthetic auxotrophs leads to adaptation to lower BipA concentration rather than escape. (A) Timeline for continuous evolution, with detection limits for escape frequency assays shown in parentheses. (B) Concentration of BipA provided to cells during evolution over a 90-day period. (C) Doubling times of progenitor and evolved synthetic auxotrophs as a function of BipA concentration, normalized to the doubling time of DEP at $100 \mu \mathrm{M}$ BipA.

genes, all of which are implicated in molecular transport: acrB. emrD, and trkH (Fig. 3A). AcrB TrkH is a potassium ion transporter ${ }^{29}$. These exact mutations have no precedent in the literature to our knowledge. Because they are missense mutations or in-frame deletions, it is unclear whether they cause loss of function or altered function (Table S1). Because permissive media contains four artificial targets of efflux (BipA, L-arabinose, chloramphenicol, and SDS), mutations that confer a selective advantage during continuous evolution could disable BipA/Larabinose efflux, improve chloramphenicol/SDS efflux, or affect transport of these or other species more indirectly. Given the strong selective pressure enforced by decreasing BipA concentration, we hypothesize that mutations observed are more likely to affect BipA transport. We also observed mutations in all evolved populations to the $23 \mathrm{~S}$ ribosomal RNA (rRNA) gene rrlA (Table S2). 23S rRNA mutations have been found to enhance tolerance for D-amino acids ${ }^{30}$ and $\beta$-amino acids ${ }^{31}$. However, 23S rRNA mutations could also be related to increased tolerance of chloramphenicol ${ }^{32}$.

To learn how identified transporter alleles may contribute to increased growth rates at low BipA concentration, we performed allelic reconstruction in the progenitor DEP strain using multiplexed automatable genome engineering (MAGE) $)^{33}$. Among four single mutants that we generated in DEP, which included both observed emrD mutant alleles, we observed growth of all mutants rather than DEP at $2 \mu \mathrm{M}$ BipA (Fig. 3B). Furthermore, only emrD mutants exhibited near-normal growth at $1 \mu \mathrm{M}$ BipA. To investigate possible differential sensitivity of strains that 
137 contain reconstructed alleles to other media components of interest (SDS, L-arabinose, Tris

138 buffer, and Chloramphenicol), we varied the concentration of these components and measured

139 doubling times (Fig. S7). We observed no significant deviation in doubling time from DEP in

140 any of these cases. These results collectively suggest that observed transporter alleles are linked

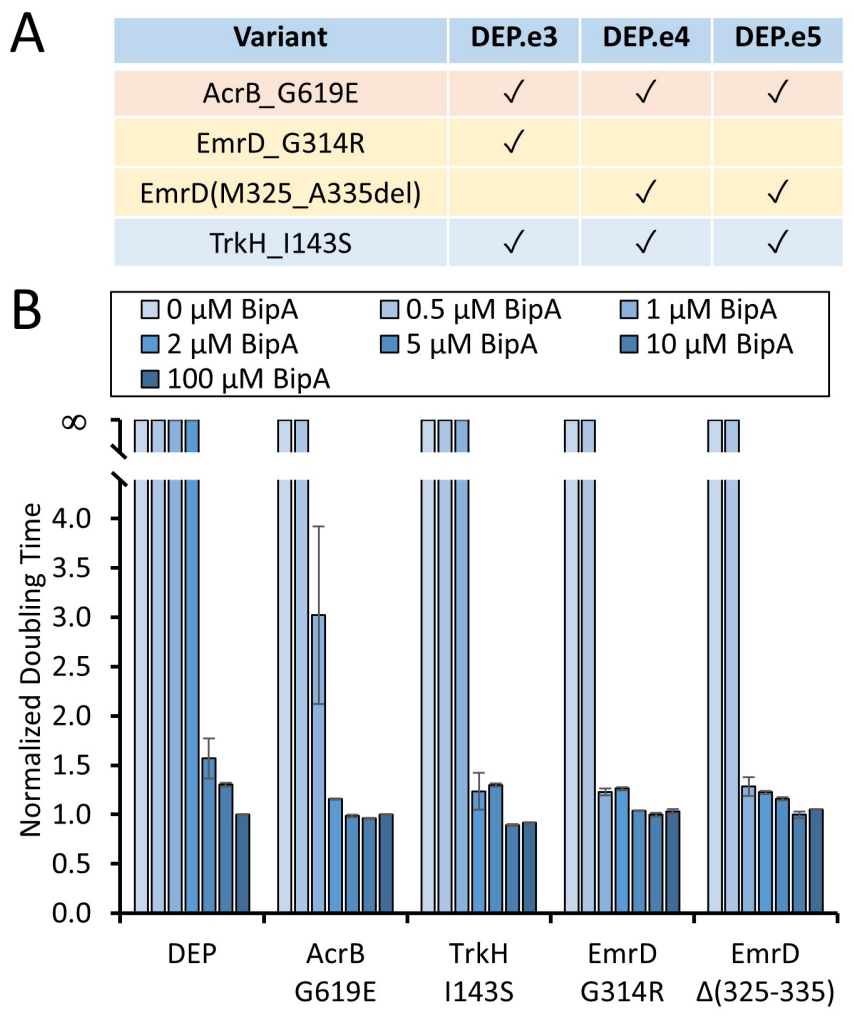

Figure 3. Whole genome sequencing and reconstruction of alleles shared across populations of evolved synthetic auxotrophs. (A) List of alleles identified through nextgeneration sequencing. (B) Effect of reconstructed allele in DEP progenitor on doubling time as a function of BipA concentration.

141 to BipA utilization.

142

\section{Bacterial-mammalian co-culturing}

The unobservable escape of DEP even after 100 days of evolution encouraged us to explore the possibility of an improved in vitro model for host-microbe interactions. In vitro models allow direct visualization and measurement of cells and effectors during processes such as pathogenesis ${ }^{34}$. They are more relevant than animal studies for several human cell-specific interactions due to biological differences across animal types ${ }^{35,36}$. A non-pathogenic $E$. coli strain engineered to express heterologous proteins could be particularly useful for studying or identifying virulence factors and disease progression. However, an obstacle associated with co- 
culture of microbial and mammalian cells is microbial takeover of the population. Approaches used to address this are bacteriostatic antibiotics ${ }^{37}$, semi-permeable Transwell membranes ${ }^{38-40}$, microcarrier beads $^{41}$, microfluidic cell-trapping ${ }^{42}$, peristaltic microfluidic flow $^{43,44}$, and microfluidic perfusion ${ }^{45}$. However, the use of a well-characterized synthetic auxotroph capable of limited persistence could offer a superior alternative for spatiotemporal control of microbial growth, especially for studying longer duration phenomena such as chronic infection or wound healing.

We investigated mammalian cell culture health, growth, and morphology after simple transient exposure to a hypermutator variant of DEP that we engineered by inactivating mutS during allelic reconstruction (DEP*). The use of DEP* rather than DEP is yet another form of a stress test to increase opportunity for escape under co-culture conditions. We directly co-cultured adherent human cell line HEK293T with either no bacteria, non-auxotrophic E. coli DH5 $\alpha$, or DEP* overnight (24 hours). HEK293T cells were cultured in selection media that allow only growth of desired but not contaminant strains while selecting for bacterial plasmid maintenance. After co-culture, we washed cells, and replenished cells with media varying in inclusion of BipA and/or an antibiotic cocktail (Penicillin/Streptomycin/Amphotericin B). We continued incubation and imaged cells at Days 2, 4, and 7 after initial co-incubation. HEK293T cells contain a copy of mCherry integrated into the AAVS1 locus and they appear red. DH5 $\alpha$ and DEP* were transformed with Clover green fluorescent protein prior to co-culture and appear green.

Compared to the control culture where bacteria were not added (Fig. 4A), HEK293T cells co-cultured with DH5 $\alpha$ display visible bacterial lawns with no attached human cells in the absence of the antibiotic cocktail at all days of observation (Fig. 4B). In the presence of antibiotic, co-cultures containing DH5 $\alpha$ sharply transition from bacterial overgrowth to apparent bacterial elimination (Fig. 4C). In contrast, cells co-cultured with DEP* in the absence of BipA exhibited similar morphology to the control at all days of observation and no detectable bacteria by fluorescence microscopy on Day 7, without the need for antibiotics to achieve bacterial clearance (Fig. 4D). Thus, DEP* addition was not detrimental to HEK293T cells in the absence of BipA and DEP* remains biocontained and cannot survive because of cross-feeding. Clearance of bacterial cells from human cells appears to occur faster for DEP* when not provided BipA (Fig. 4D) than for DH5 $\alpha$ when provided the antibiotic cocktail (Fig 4C). 


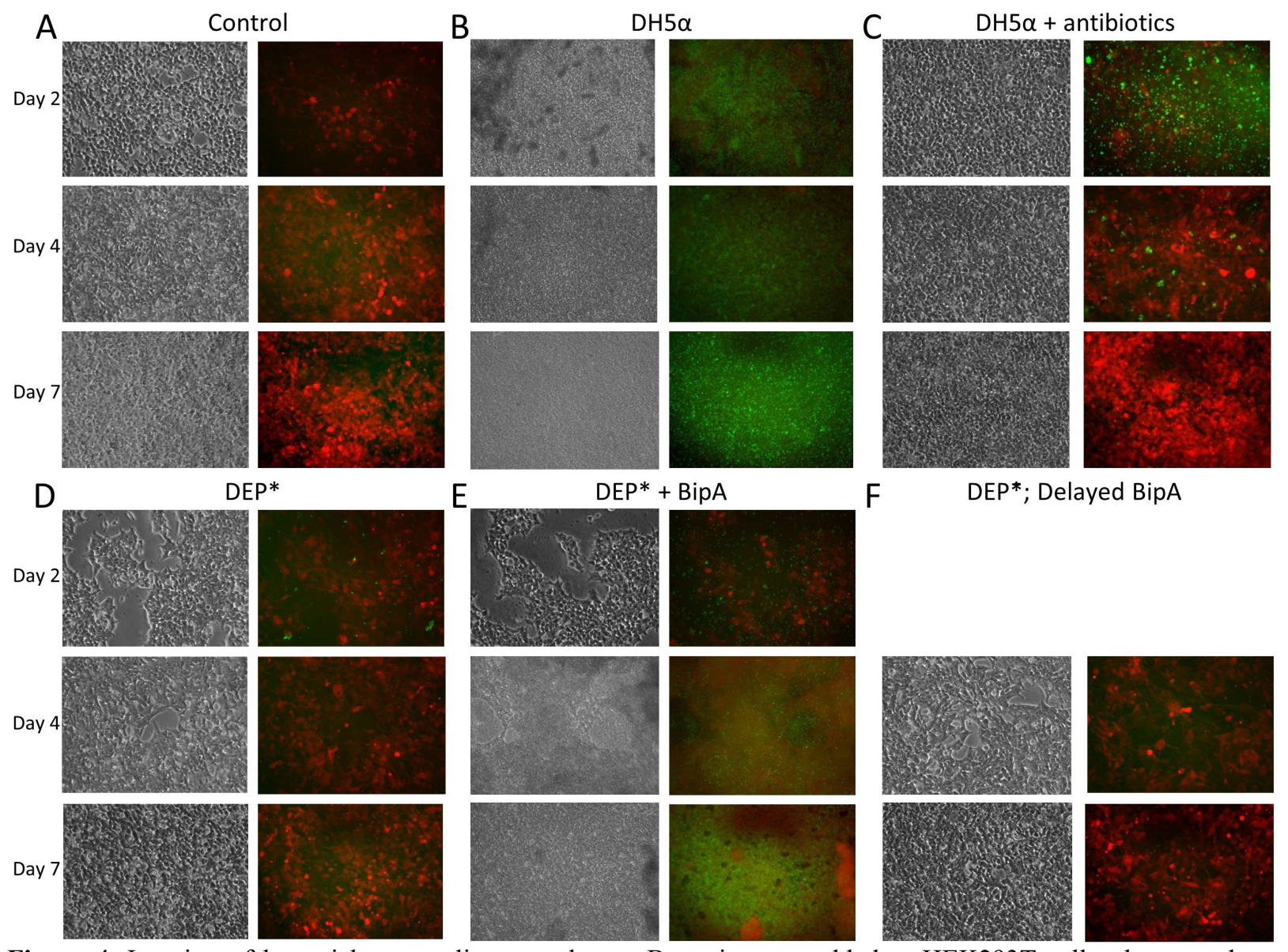

Figure 4. Imaging of bacterial-mammalian co-cultures. Bacteria were added to HEK293T cell cultures and coincubated for 24 hours before washing and replenishing media. HEK293T cells express mCherry, whereas bacterial cells express Clover green protein marker. Images were taken at Days 2, 4, and 7 after co-incubation. (A) Untreated HEK293T cells. (B) HEK293T with commercial E. coli DH5a in the absence of antibiotic cocktail. (C) HEK293T with DH5 $\alpha$ in presence of antibiotic cocktail. (D) HEK293T and DEP* (mismatch repair inactivated to create hypermutator phenotype) in the absence of BipA. (E) HEK293T cells and DEP* in the presence of BipA. (F) HEK293T and DEP* in the absence of BipA until Day 2 (identical at this point to condition in Panel D), and then $100 \mu \mathrm{M}$ of BipA added to this condition daily until Day 7.

To learn how the synthetic auxotroph behaves when supplied its essential nutrient in these co-culture settings, we tested DEP* co-cultures with continual resupply of $100 \mu \mathrm{M}$ BipA. Here, DEP* proliferates and in turn decreases proliferation and viability of HEK293T cells (Fig. 4E). A bacterial lawn begins to form on Day 2 and at later times human cell debris is overtaken by DEP*. This demonstrates that DEP* is fully capable of taking over the co-culture if supplied with BipA. Replicates for these experiments can be found in Figs. S8-10.

Given that DEP* grows in co-cultures when BipA is provided, we sought to understand whether it could be rescued by re-addition of BipA after multiple days of withholding. The possible timescale of re-emergence influences applications where the duration of bacterial 
activity would need to be prolonged and/or repeated via limited BipA introduction while remaining contained. We find that co-culturing DEP* with HEK293T cells for 2 days in absence of BipA followed by addition of BipA at Day 2 does not rescue the DEP* growth (Fig. 4 F and S11). Human cells still grow and look morphologically similar to untreated cells and bacteria are not visible. To look at analogous questions for non-auxotrophic E. coli, we removed antibiotics after 2 days of co-culturing and do not observe bacterial rescue (Fig. S11). We also investigated whether bacterial clearance could be delayed by addition of antibiotic after some growth of DH5 $\alpha$. DH5 $\alpha$ cells grown in absence of the antibiotic cocktail for 2 days before addition of the cocktail and maintenance to Day 7 result in bacterial lawns (Fig. S11A and D). This demonstrates that antibiotic cocktails ordinarily used in mammalian cell culture maintenance can become ineffective beyond a certain amount of non-auxotrophic bacterial growth, whereas synthetic auxotrophy is subject to fewer and different constraints.

To further investigate the persistence of progenitor DEP and its evolved descendants, we performed BipA re-addition studies in LB monoculture. Within 7 hours of BipA removal, DEP cell populations that are harvested from mid-exponential or stationary phases can be "reactivated" upon delayed BipA addition with unperturbed growth kinetics after a highly tunable lag phase (Fig. S12). Growth curve and colony count experiments for cells deprived of BipA for longer timescales demonstrate that growth can be recovered upon BipA reintroduction after as long as 48 hours under these monoculture conditions (Figs. S13-14).

We have shown that synthetic auxotrophy can exhibit long-term stability and function in unique contexts, enabling reliable control of microbial proliferation. Recent work has also shown that the escape rate and fitness of multiple synthetic auxotrophs can be improved by increasing the specificity of nsAA incorporation machinery ${ }^{46}$. Collectively, these engineering and characterization efforts advance synthetic auxotrophy as a powerful safeguard for basic and applied research when using engineered microbes.

\section{Acknowledgements:}

We thank Caleb Bashor for assistance setting up the continuous evolution platform, and Sabyasachi Sen and Neil Butler for manuscript comments. We thank Timothy Wannier for sharing the Clover plasmid, and Ellen Schrock for helping generate the mCherry stable HEK293T cell line. This work was primarily funded by grants to GMC from the U.S. Dept. of 
221 Energy (FG02-02ER63445) and the FunGCAT program from the Office of the Director of 222 National Intelligence (ODNI), Intelligence Advanced Research Projects Activity (IARPA), via 223 the Army Research Office (ARO) under Federal Award No. W911NF-17-2-0089. We also 224 acknowledge support from the National Institute of General Medical Sciences of the National 225 Institutes of Health under a Chemistry-Biology Interface Training Grant that supported MAJ 226 (Award Number T32GM133395), and a grant to AMK from the National Science Foundation 227 (MCB2027092).

228 The authors declare the following competing financial interest(s): GMC has related 229 financial interests in 64-x, EnEvolv, and GRO Biosciences. For a complete list of GMC's 230 financial interests, please visit arep.med.harvard.edu/gmc/tech.html. 


\section{References:}

1. Parker, M. T. \& Kunjapur, A. M. Deployment of Engineered Microbes: Contributions to the Bioeconomy and Considerations for Biosecurity. Heal. Secur. 18, 278-296 (2020).

2. Bober, J. R., Beisel, C. L. \& Nair, N. U. Synthetic Biology Approaches to Engineer Probiotics and Members of the Human Microbiota for Biomedical Applications. Annu. Rev. Biomed. Eng. 20, 277-300 (2018).

3. Ozdemir, T., Fedorec, A. J. H., Danino, T. \& Barnes, C. P. Synthetic Biology and Engineered Live Biotherapeutics: Toward Increasing System Complexity. Cell Systems 7, 5-16 (2018).

4. Charbonneau, M. R., Isabella, V. M., Li, N. \& Kurtz, C. B. Developing a new class of engineered live bacterial therapeutics to treat human diseases. Nat. Commun. 11, 1738 (2020).

5. Bacon, G. A., Burrows, T. W. \& Yates, M. The effects of biochemical mutation on the virulence of Bacterium typhosum; the loss of virulence of certain mutants. Br. J. Exp. Pathol. 32, 85-96 (1951).

6. Hoiseth, S. K. \& Stocker, B. A. D. Aromatic-dependent Salmonella typhimurium are nonvirulent and effective as live vaccines. Nature 291, 238-239 (1981).

7. Thompson, R. J., Bouwer, H. G., Portnoy, D. A. \& Frankel, F. R. Pathogenicity and immunogenicity of a Listeria monocytogenes strain that requires D-alanine for growth. Infect. Immun. 66, 3552-61 (1998).

8. Smith, D. A., Parish, T., Stoker, N. G. \& Bancroft, G. J. Characterization of auxotrophic mutants of Mycobacterium tuberculosis and their potential as vaccine candidates. Infect. Immun. 69, 1142-1150 (2001).

9. Hinchey, J. et al. Lysine auxotrophy combined with deletion of the secA2 gene results in a safe and highly immunogenic candidate live attenuated vaccine for tuberculosis. PLoS One 6, (2011).

10. Cabral, M. P. et al. Design of live attenuated bacterial vaccines based on D-glutamate auxotrophy. Nat. Commun. 8, 15480 (2017).

11. Tjuvajev, J. et al. Salmonella-based tumor-targeted cancer therapy: Tumor amplified protein expression therapy (TAPET ${ }^{\mathrm{TM}}$ ) for diagnostic imaging. in Journal of Controlled Release 74, 313-315 (Elsevier, 2001). 
12. Steidler, L. et al. Biological containment of genetically modified Lactococcus lactis for intestinal delivery of human interleukin 10. Nat. Biotechnol. 21, 785-789 (2003).

13. Zhao, M. et al. Tumor-targeting bacterial therapy with amino acid auxotrophs of GFPexpressing Salmonella typhimurium. Proc. Natl. Acad. Sci. U. S. A. 102, 755-760 (2005).

14. Braat, H. et al. A Phase I Trial With Transgenic Bacteria Expressing Interleukin-10 in Crohn's Disease. Clin. Gastroenterol. Hepatol. 4, 754-759 (2006).

15. Leventhal, D. S. et al. Immunotherapy with engineered bacteria by targeting the STING pathway for anti-tumor immunity. Nat. Commun. 11, 2739 (2020).

16. Formal, S. B., Baron, L. S. \& Spilman, W. Studies on the virulence of a naturally occurring mutant of Salmonella typhosa. J. Bacteriol. 68, 117 (1954).

17. Curcho, M. D. L. G. Mutation to Tryptophan Independence in Eberthella typhosa. J. Bacteriol. 56, 374 (1948).

18. Fildes, P. \& Whitaker, K. "Training” or Mutation of Bacteria. Br. J. Exp. Pathol. 29, 240 (1948).

19. Liu, S. C., Minton, N. P., Giaccia, A. J. \& Brown, J. M. Anticancer efficacy of systemically delivered anaerobic bacteria as gene therapy vectors targeting tumor hypoxia/necrosis. Gene Ther. 9, 291-296 (2002).

20. Anderson, J. C., Clarke, E. J., Arkin, A. P. \& Voigt, C. A. Environmentally controlled invasion of cancer cells by engineered bacteria. J. Mol. Biol. 355, 619-627 (2006).

21. Pande, S. et al. Metabolic cross-feeding via intercellular nanotubes among bacteria. Nat. Commun. 6, 6238 (2015).

22. Zengler, K. \& Zaramela, L. S. The social network of microorganisms - How auxotrophies shape complex communities. Nature Reviews Microbiology 16, 383-390 (2018).

23. Mandell, D. J. et al. Biocontainment of genetically modified organisms by synthetic protein design. Nature 518, 55-60 (2015).

24. Rovner, A. J. et al. Recoded organisms engineered to depend on synthetic amino acids. Nature 518, 89-93 (2015).

25. Wannier, T. M. et al. Adaptive evolution of genomically recoded Escherichia coli. Proc. Natl. Acad. Sci. U. S. A. 115, (2018).

26. Wong, B. G., Mancuso, C. P., Kiriakov, S., Bashor, C. J. \& Khalil, A. S. Precise, automated control of conditions for high-throughput growth of yeast and bacteria with 
eVOLVER. Nat. Biotechnol. 36, 614-623 (2018).

27. Toprak, E. et al. Building a morbidostat: an automated continuous-culture device for studying bacterial drug resistance under dynamically sustained drug inhibition. Nat. Protoc. 8, 555-67 (2013).

28. Nishino, K. \& Yamaguchi, A. Analysis of a complete library of putative drug transporter genes in Escherichia coli. J. Bacteriol. 183, 5803-5812 (2001).

29. Levin, E. J. \& Zhou, M. Recent progress on the structure and function of the TrkH/KtrB ion channel. Current Opinion in Structural Biology 27, 95-101 (2014).

30. Dedkova, L. M., Fahmi, N. E., Golovine, S. Y. \& Hecht, S. M. Construction of modified ribosomes for incorporation of D-amino acids into proteins. Biochemistry 45, 1554115551 (2006).

31. Maini, R. et al. Protein Synthesis with Ribosomes Selected for the Incorporation of $\beta$ Amino Acids. Biochemistry 54, 3694-3706 (2015).

32. Ettayebi, M., Prasad, S. M. \& Morgan, E. A. Chloramphenicol-erythromycin resistance mutations in a 23S rRNA gene of Escherichia coli. J. Bacteriol. 162, 551-557 (1985).

33. Wang, H. H. et al. Programming cells by multiplex genome engineering and accelerated evolution. Nature 460, 894-898 (2009).

34. Goers, L., Freemont, P. \& Polizzi, K. M. Co-culture systems and technologies: taking synthetic biology to the next level. J R Soc Interface 11, (2014).

35. Nguyen, T. L. A., Vieira-Silva, S., Liston, A. \& Raes, J. How informative is the mouse for human gut microbiota research? DMM Dis. Model. Mech. 8, 1-16 (2015).

36. Paul, W., Marta, C. \& Tom, V. de W. Resolving host-microbe interactions in the gut: the promise of in vitro models to complement in vivo research. Current Opinion in Microbiology 44, 28-33 (2018).

37. Mason, K. M., Bigley, N. J. \& Fink, P. S. Development of a novel in vitro co-culture system for studying host response to native bacterial antigens. J. Immunol. Methods 211, 147-158 (1998).

38. Parlesak, A., Haller, D., Brinz, S., Baeuerlein, A. \& Bode, C. Modulation of Cytokine Release by Differentiated CACO-2 Cells in a Compartmentalized Coculture Model with Mononuclear Leucocytes and Nonpathogenic Bacteria. Scand. J. Immunol. 60, 477-485 (2004). 
39. Nuenen, M. H. M. C. et al. The influence of microbial metabolites on human intestinal epithelial cells and macrophages in vitro. FEMS Immunol. Med. Microbiol. 45, 183-189 (2005).

40. Noel, G. et al. A primary human macrophage-enteroid co-culture model to investigate mucosal gut physiology and host-pathogen interactions. Sci. Rep. 7, 1-14 (2017).

41. Höner zu Bentrup, K. et al. Three-dimensional organotypic models of human colonic epithelium to study the early stages of enteric salmonellosis. Microbes Infect. 8, 18131825 (2006).

42. Kim, J., Hegde, M. \& Jayaraman, A. Co-culture of epithelial cells and bacteria for investigating host-pathogen interactions. Lab Chip 10, 43-50 (2010).

43. Kim, H. J., Huh, D., Hamilton, G. \& Ingber, D. E. Human gut-on-a-chip inhabited by microbial flora that experiences intestinal peristalsis-like motions and flow. Lab Chip 12, 2165-2174 (2012).

44. Kim, H. J., Li, H., Collins, J. J. \& Ingber, D. E. Contributions of microbiome and mechanical deformation to intestinal bacterial overgrowth and inflammation in a human gut-on-a-chip. Proc. Natl. Acad. Sci. U. S. A. 113, E7-E15 (2016).

45. Shah, P. et al. A microfluidics-based in vitro model of the gastrointestinal human-microbe interface. Nat. Commun. 7, 11535 (2016).

46. Kunjapur, A. M. et al. Engineering posttranslational proofreading to discriminate nonstandard amino acids. Proc. Natl. Acad. Sci. U. S. A. 115, 619-624 (2018). 